\title{
Collapse of 4D random geometries
}

\author{
P. Bialas Z. Burda
}

October 19, 2018

\begin{abstract}
We extend the analysis of the Backgammon model to an ensemble with a fixed number of balls and a fluctuating number of boxes. In this ensemble the model exhibits a first order phase transition analogous to the one in higher dimensional simplicial gravity. The transition relies on a kinematic condensation and reflects a crisis of the integration measure which is probably a part of the more general problem with the measure for functional integration over higher $(d>2)$ dimensional Riemannian structures.
\end{abstract}

Recently we have proposed a scenario for the phase transition in higher dimensional simplicial quantum gravity based on the balls-in-boxes model [1, 2] also called Backgammon model in a different context [3]. It has been established that simplicial gravity has two phases: crumpled and elongated. In the elongated phase it is effectively described by the Branched Polymer model 四. In the crumpled phase is characterized by the appearance of a singular vertices which gather around them an extensive part of the volume [5, [0]. The transition between those two phases is of first order [7, 8]. The constrained-mean-field interpretation based on Backgammon model captures all those features except the order of the transition.

In this letter we show that the modified balls-in-boxes model undergoes a first order phase transition of the same type as in simplicial gravity.

We start with a brief review of the properties the Backgammon model. The model describes $N$ balls distributed in $M$ boxes. The partition function reads [2, 3]:

$$
Z_{M, N}=\sum_{q_{1}, \ldots, q_{M}} p\left(q_{1}\right) \cdots p\left(q_{M}\right) \delta_{q_{1}+\cdots+q_{M}, N}
$$

The numbers of balls $q_{i}$ and $q_{j}$ in two boxes $i$ and $j$ are independent as reflected by the factorization of the total thermodynamic weight to one-box 
weights $p(q)$ 's. The factorization is weakly broken by the constrain on the total number of balls. This constrain introduces correlations between boxes and may result in the condensation of balls into one box [2].

We are interested in the limit of $N \rightarrow \infty$ and $r=M / N$ fixed. We call the quantity $r$ curvature. In this limit one expects the partition function to behave as

$$
Z_{M, N} \sim \exp (N f(r)+\cdots)
$$

where the dots stand for corrections growing slower than linearly in $N$. The function $f(r)$ is the free energy density per ball. The quantity $r$ is bounded from below by $1 / N$ since the system has to have at least one box. Additionally to fix attention we introduce an upper bound on $r$ by requiring that each box must contain at least one ball. These give together : $1 / N \leq r \leq 1$.

The phase structure of the model is encoded in the analytic properties of the function (the log does not change those properties and is added for convenience):

$$
K(\mu)=-\log \sum_{q} p(q) e^{-\mu q}
$$

In particular if the series under the logarithm has a finite radius of convergence then the model given by the partition function (11) has a very interesting phase structure [2, 9]. Denote the real value of $\mu$ at the radius of convergence by $\mu_{c r}$, and restrict ourselves to the situation when the value of the derivative $K^{\prime}\left(\mu_{c r}\right)$ is finite as happens for instance for the weights of the form $p(q)=q^{-\alpha} e^{-\mu_{c r} q}$ when $\alpha>2$. In this case it can be shown that the system has two phases depending on the value of curvature $r$ [2].

The free energy density $f(r)$ (2) can be found by the steepest descent method. One obtains the following expression :

$$
f(r)= \begin{cases}-r K\left(\mu_{*}(r)\right)+\mu_{*}(r) & \text { for } r>r_{c r} \\ -r \kappa_{c r}+\mu_{c r} & \text { for } r \leq r_{c r}\end{cases}
$$

where $\mu_{*}(r)$ is given by the solution of the saddle point equation

$$
\frac{1}{r}=K^{\prime}\left(\mu_{*}\right)
$$

\footnotetext{
${ }^{1}$ One might alternatively formulate the limit in terms of $M$ and $\rho=N / M: Z_{M, N} \sim$ $\exp (M f(\rho)+\cdots)$ as in [2]. In fact this would look more natural, since $\rho$ has an interpretation of the average number of balls per box and not the number of boxes per ball as defined by $r$. Moreover it would be more natural to keep $M$ in front of $f$ since then $f$ had an interpretation of the free energy density per box. We use the convention with $r$ and $N$ on purpose since it is better suited for calculations in the ensemble with varying $M$ which is the subject of the letter.
} 
The positivity of the weights $p(q)$ 's implies that the function on the right hand side of (5) is monotonic. From this follows that the solution $\mu_{*}$ is unique and that it is a monotonic real function of $r$. For $r=1$ it is infinite. When $r$ decreases so does $\mu_{*}$. The equation has a solution for $\mu_{*}$ as long as $r$ is larger then a critical value $r_{c r}$. The critical value $r_{c r}$ is obtained from the equation (5) when on the right hand side $\mu_{*}=\mu_{c r}$ reaches the singularity of $K$. At the critical value the free energy is given by the formula in the lower line of (4) where $\kappa_{c r}=K\left(\mu_{c r}\right)$ and sticks to this value even when one $r$ is lower since $\mu_{*}$ may not move beyond $\mu_{c r}$. At this point the system undergoes a continuous phase transition [2].

In simplicial gravity the analog of the quantity $r$ is the ratio $N_{0} / N_{4}$ where the $N_{0}$ is the number of vertices and $N_{4}$ is the number of 4 -simplices. The vertices play the role of boxes and the 4 -simplices that of balls. The number of balls in one box is analogue of the number of vertices sharing one vertex $i e$ vertex order. The sum of vertex orders $q_{i}$ is constrained by the relation: $q_{1}+\cdots+q_{N_{0}}=5 N_{4}$. The ratio $N_{0} / N_{4}$ is a linear function of Regge curvature. The average curvature is varied through a coupling to the number of vertices.

In this letter we study the model with fluctuating number of boxes which more closely resembles the simplicial gravity scenario. The partition function of this model is :

$$
Z(\kappa, N)=\sum_{M=1}^{N} Z_{M, N} e^{\kappa M}
$$

Substituting $Z_{M, N}$ by (2) we get in the large $N$ limit :

$$
Z(\kappa, N)=N \int_{0}^{1} \mathrm{~d} r \exp N(\kappa r+f(r))
$$

where $f(r)$ has the form (4). The integrand is the distribution of the curvature for a given $\kappa$. For large $N$ one expects the integrand to approach gaussian shape centered around $r_{*}$ being the solution of the saddle point equation :

$$
\kappa+f^{\prime}\left(r_{*}\right)=0
$$

For $\kappa>\kappa_{c r}$ this equation reduces to $\kappa=K\left(\mu_{s p}\left(r_{*}\right)\right)$ which has a unique solution for $r_{*}$. The value of $r_{*}$ being the position of the gaussian distribution is the average curvature in the limit $N \rightarrow \infty$. This situation continues as long as $\kappa>\kappa_{c r}$.

Below the critical point $\kappa<\kappa_{c r}$ the free energy $f(r)$ is linear in $r$ and the saddle point equation (8) has no solution. The integrand is not gaussian anymore but a decreasing function of $r$. It is exponential : $\exp N\left(\kappa-\kappa_{c r}\right) r$ 


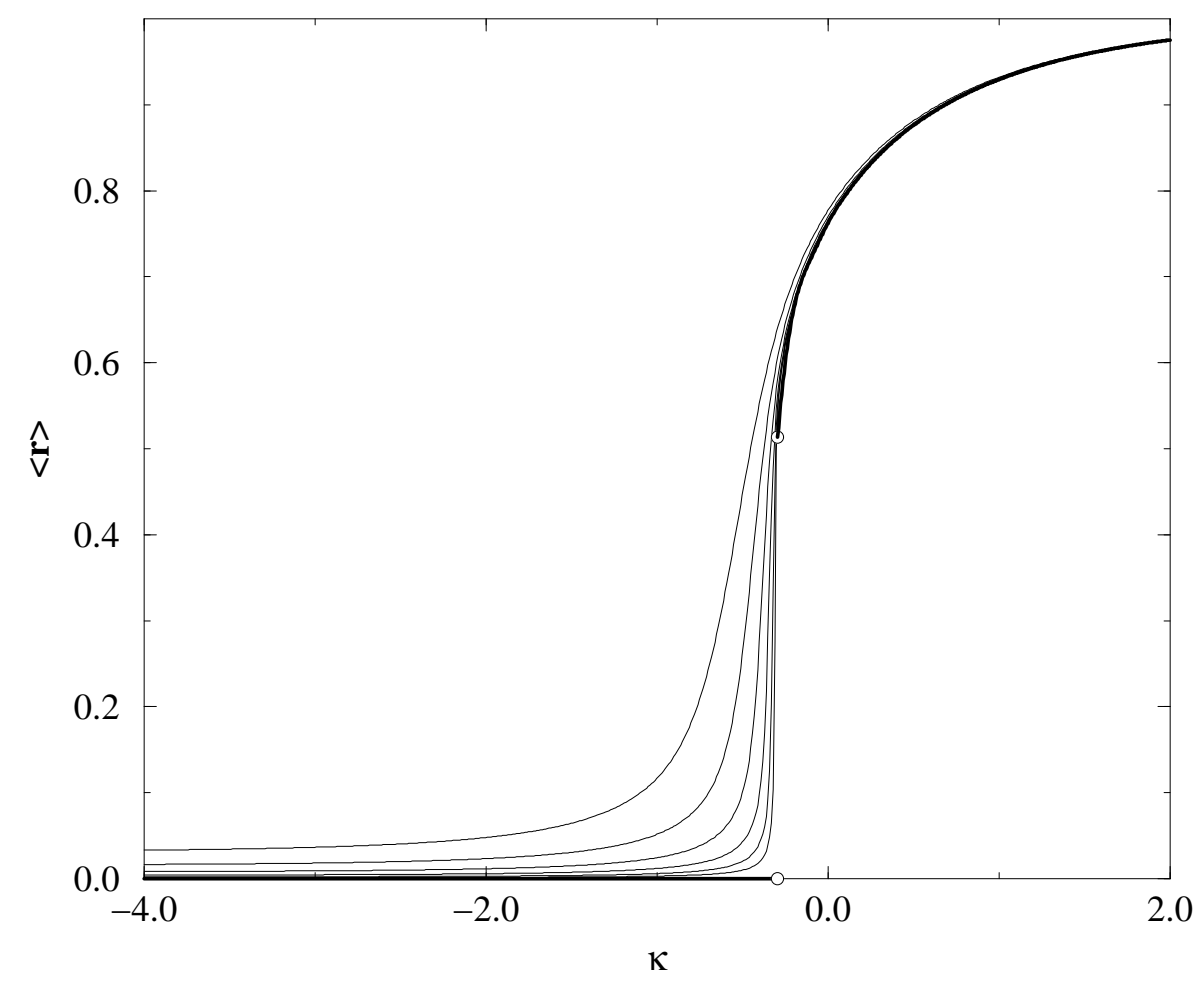

Figure 1: Average curvature as the function of $\kappa$ for infinite N (bold line) 32, 64, 128, 256, 512 and 1024 balls

for $r<r_{c r}$. For large $N$ only this exponential part matters in the integral (7) and one finds that $\langle r>\sim 1 / N$.

Exactly at the critical point $\kappa=\kappa_{c r}$, the equation (8) is fulfilled by all $r$ between 0 and $r_{c r}$ as can be seen from the second line of (何). The intuition behind this is that the curvature $r$ must abruptly move between two separated regimes, namely gaussian at $r=r_{c r}$ and exponential at $r=0$. Therefore $r$ stays undetermined by the saddle point equation. To fix the shape of the integrand one has to consider finite $N$ systems.

In the figure 1 we show average curvature $\langle r\rangle$ as a function of $\kappa$ for weights $p(q)=q^{-\frac{5}{2}}$. For $\kappa>\kappa_{c r}$ it is a solution of the saddle point equation (8). It stops at $r_{c r}$ and falls to zero. In the same figure we plot average curvature for some finite values of $N$. The results were obtained by a recursive technique described in [2]. In the gaussian phase $\kappa>\kappa_{c r}$ the curves lie close to each other indicating that the finite size effects are small there. For large $\kappa$ the curves approach asymptotically the upper kinematic bound $r=1$. In the exponential (kinematic) phase $\kappa<\kappa_{c r}$ finite size effects are stronger reflecting 


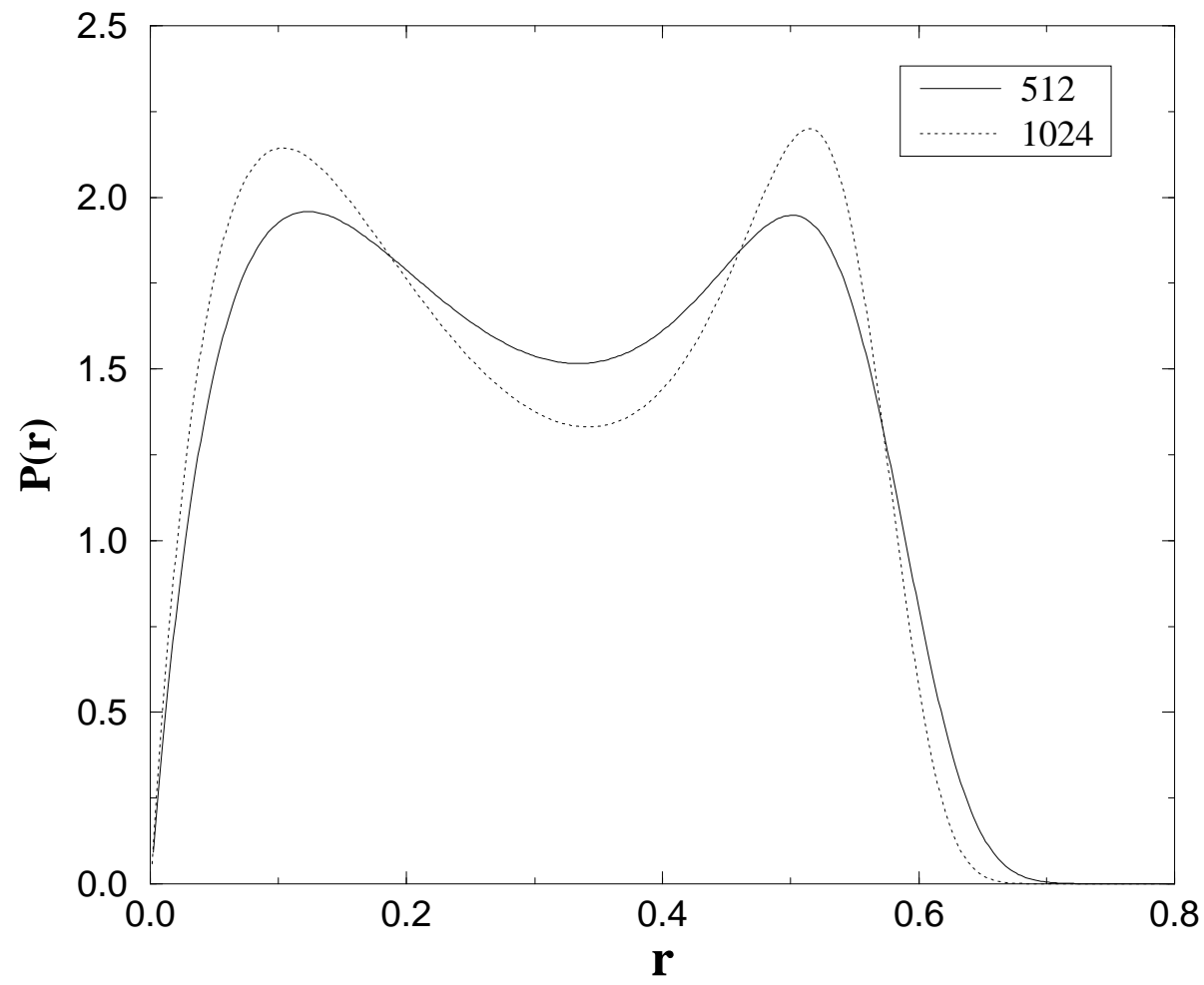

Figure 2: Distribution of the curvature for $512(\kappa=-0.32184)$ and 1024 $(\kappa=-0.31910)$ near phase transition

the size dependence of the lower kinematic bound $1 / N$. This also agrees with the results of numerical simulations of simplicial gravity, but there the kinematic bound is $1 / \sqrt{N}$ rather than $1 / N$.

The steepest part of the curves corresponds to the pseudocritical region. It is a crossover region where the two phases coexist. The spread of the pseudocritcial region is $\delta \kappa \sim 1 / N$ and reduces to one point for infinite $N$. In the figure 2 we show the distributions of $r$ for $N=512$ and $N=1024$. One sees coexistence of two phases. The fraction of either phase depends on a particular choice of $\kappa$ in the pseudocritical region. We picked its values in such a way as to have more or less the same heights of both the peaks. The similar two peak structure has been observed in simplicial gravity for systems with 32000 and 64000 simplices [7, 8]

This nicely extends the results of [1] showing that all the thermodynamical (non-geometrical) features of the simplicial gravity can be explained by this very simple model. It seems that the mean field sets in already for $d \geq 3$. The phase transition from Branched Polymer phase to the crumpled 
phase is kinematical $i e$ it is associated with system approaching the phase space boundary rather then diverging correlations (interactions). We think that this reflects the crisis of the measure in higher dimensional geometries. However it may also be that the gravity does not exist alone and needs some matter fields which would locally smooth the manifold and open a physical window. Unfortunately, fermions on random lattice seem to be for the time being far beyond numerical access.

Of course the presented model can only capture the bulk features of the system as all the geometry has been integrated out, so its simplicity does not exclude the possibility of interesting geometrical behavior. One simple geometrical realization of Bacgammon is BP model [a]. In this model exactly at the phase transition system enters a third phase which has a different Hausdorff dimension [9, 10]. It may be that the same phenomenon happens in simplicial gravity.

The authors thanks Desmond Johnston and Jerzy Jurkiewicz for helpful comments and discussion. This work was partially supported by KBN grants 2P03B19609.

\section{References}

[1] P. Bialas, Z. Burda, B. Petersson, J. Tabaczek Nucl. Phys. B495 (1997) 463.

[2] P. Bialas, Z. Burda, D. Johnston Nucl. Phys. B493 (1997) 505.

[3] F. Ritort, Phys. Rev. Lett. 75 (1995) 1190

F. Ritort and S. Franz, Europhys Lett. 31 (1995) 507, condmat/9505115;

F. Ritort and S. Franz, "Glassy mean field behaviour of the backgammon model", cond-mat/9508133;

C. Godreche, J.P. Bouchaud and M. Mezard, J. Phys A28, (1995) L603;

C. Godreche and J.M. Luck, J. Phys A29 (1996) 1915.

[4] J. Ambjørn, J. Jurkiewicz, Nucl. Phys. B541 (1995) 643.

[5] T.Hotta, T. Izubuchi, J. Nishimura, Nucl. Phys B (Proc. Suppl.) 47 (1995) 609.

[6] S. Cateral, J. Kogut, R. Renken, G. Thorleifsson, Nucl. Phys. B468 (1996) 263.

[7] P. Bialas, Z. Burda, A. Krzywicki, B. Petersson, Nucl. Phys. B472, (1996) 293. 
[8] B. V. de Bakker Phys. Lett. B389 (1996) 238.

[9] P. Bialas, Z. Burda, Phys. Lett. B384(1996) 75.

[10] J. Jurkiewicz, A. Krzywicki Phys. Lett. B392(1997) 291. 